\title{
Progress towards the Millennium Development Goals in SA
}

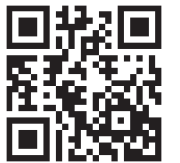

The Millennium Declaration, together with the Millennium Development Goals (MDGs), ${ }^{[1]}$ was signed by member states of the United Nations, including South Africa (SA), in September 2000. While all the MDGs are related to health, goals 4,5 and 6 are directly related to health and the functioning of the healthcare system: goal 4 relates to reducing child mortality, goal 5 to improving maternal health, and goal 6 to combating HIV/AIDS, malaria and other diseases, such as tuberculosis (TB). Targets were set for countries to reach by 31 December 2015, using the year 1990 as a baseline. This supplement contains a number of papers that describe the progress SA has made and some of the continuing challenges that need to be overcome to meet the targets, or at least get as close to reaching them as possible.

Leading the way is the objective to eliminate malaria, defined as zero malaria cases transmitted in SA by $2018{ }^{[2]}$ There has been a remarkable $90 \%$ decrease in the number of cases against the baseline of the year 2000 and a corresponding $80 \%$ reduction in mortality. For the past 5 years there have been $<10000$ cases annually and $<100$ deaths. These successes have been due to vector control through indoor residual spraying, coupled with treatment with artemesinin-based combination treatments and having good co-operation with neighbouring states, especially Mozambique, as the mosquito vector does not require a passport to cross borders! Having adopted malaria elimination as a policy, SA will need to strengthen its efforts further with specific focus on monitoring parasite drug and vector resistance, active surveillance, and utilising sensitive diagnostic tests to detect low-level parasitaemia.

In response to high childhood mortality from pneumonia and diarrhoea, in 2009 SA was the first country in Africa to introduce pneumococcal conjugate vaccine and rotavirus vaccine into its routine Expanded Programme for Immunization (EPI). Madhi et al. ${ }^{[3]}$ show that by 2012 these two vaccines had been successfully integrated into the EPI schedule and universal coverage on a par with the traditional vaccines had been achieved. Since 2008, there has been a remarkable drop in the incidence of pneumonia and diarrhoea in children under 5 years of age. This has been coupled with a halving of the case fatality rate of children who have been admitted to hospital with these two diseases. So, not only are there far fewer cases of pneumonia and diarrhoea, but those that have occurred have been less severe.

When the history of public health in SA is written, one of the chapters of success will be the remarkable achievements made in reducing mother-to-child transmission (MTCT) of HIV. The laboratory monitoring of MTCT and provision of objective hard evidence of where and how much MTCT has occurred have played key roles in this reduction. Sherman et al. ${ }^{[4]}$ highlight the annual increase in coverage of testing for MTCT and the annual decrease in transmission. By 2012 nearly three-quarters $(73 \%)$ of the estimated number of infants who had been exposed to HIV at birth were tested for transmission at around 6 weeks and $2.4 \%$ were found to be infected. The significant reduction in annual MTCT rates has played a major role in the rapid reductions in child and infant mortality rates that we have seen in SA recently. ${ }^{[5]}$

Using colour-coded dashboards and targets to pinpoint areas of success and weakness, down to district and facility levels, has also contributed to the success of the MTCT programme. Bhardwaj et al.$^{[6]}$ have highlighted how quality improvement interventions using datadriven participatory processes to understand facility-level bottlenecks have been instrumental in optimising the scale-up of national policies throughout the public healthcare system.

SA has the highest estimated incidence and prevalence of TB worldwide, due in part to the linkage with the HIV epidemic. ${ }^{[7]}$ However,

there have been a number of notable achievements over the past 5 years. These include a decrease in the number of TB cases notified annually, a decrease in the number of deaths from TB, an increase in successful treatment outcomes for new smear-positive patients, and improved prevention of TB in patients infected with HIV. The national rollout of the GeneXpert diagnostic technology, the largest in the world, is one example of a commitment to decreasing the burden of disease from TB. However, for further progress new TB drugs and vaccines are required to further accelerate progress towards improved TB control.

SA has moved from pariah status to a country with an HIV programme that has received national and international acclaim. ${ }^{[8]}$ Simelela and Venter ${ }^{[0]}$ give an overview of the response to HIV in SA. Despite many obstacles, over the past 4 years the story of the AIDS response in SA is one of great progress and the country has by far the largest antiretroviral programme in the world, with well over 2 million HIV-infected people receiving treatment. This is an example of what can be achieved when government, civil society and international partners come together and harness resources to achieve a common vision. However, the hard challenge of strengthening the healthcare system to ensure decades of effective HIV prevention and care looms ahead.

This supplement illustrates some of the many successes achieved. However, most of the low-hanging fruit has been picked and now the healthcare system has to be significantly improved to ensure that the trajectory of success towards the MDGs and beyond is maintained.

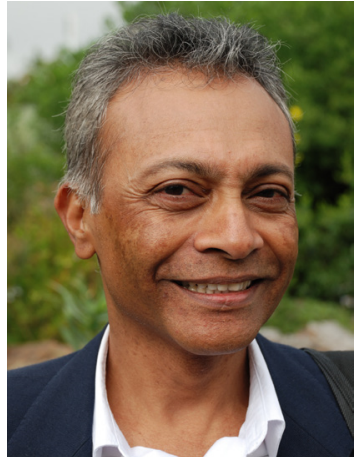

Yogan Pillay

National Department of Health,

Pretoria, South Africa

ypillay@intekom.co.za

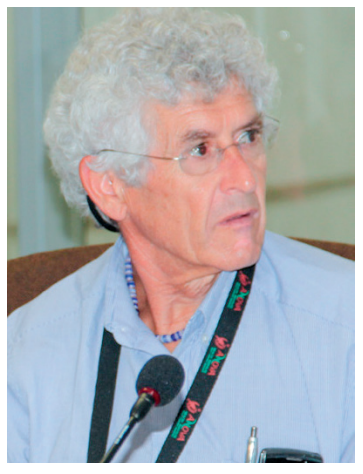

Peter Barron

School of Public Health, Faculty of Health Sciences, University of the Witwatersrand, Johannesburg, South Africa pbarron@iafrica.com

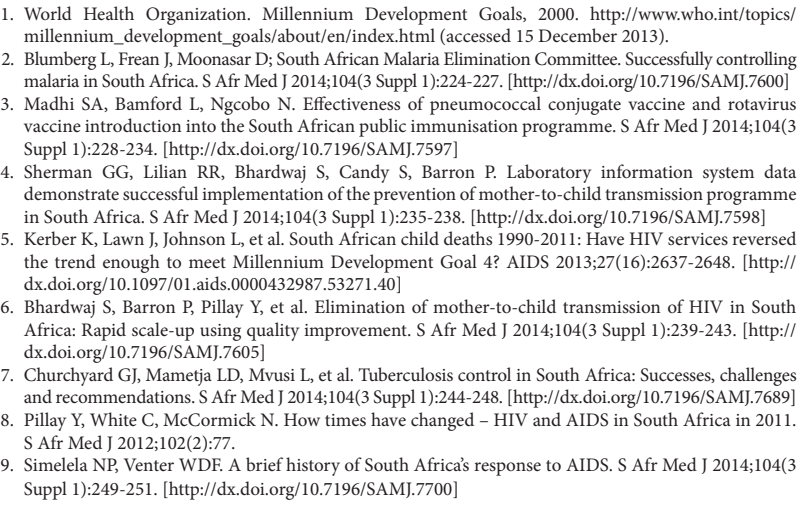

S Afr Med J 2014;104(3):223. DOI:10.7196/SAMJ.7924 